\title{
Pacific
}

Journal of

Mathematics

\section{SEMIFREE ACTIONS ON SPHERES}

\author{
MONICA NicOlaU
}

Volume $156 \quad$ No. 2

December 1992 


\title{
SEMIFREE ACTIONS ON SPHERES
}

\author{
MoNica NicolaU
}

\begin{abstract}
We employ knot theoretic techniques to construct a strong relationship between free and semifree $Z_{m}$ actions on high dimensional spheres. This correspondence is then used to study free actions on spherical codimension two links.
\end{abstract}

Introduction. The present paper deals with the problem of comparing free and semifree $\mathbf{Z}_{m}$ actions on high dimensional spheres. Our approach is to consider fixed or invariant subsets, and use equivariant knot theoretic techniques to show that, in codimension two, the classification and the existence questions are essentially the same, for free and for semifree actions. This strong correspondence is then used to study free cyclic actions on high dimensional spherical links of codimension two. We show that such links can exhibit at most one component which is itself invariant under the action, all other components being permuted.

All manifolds considered will be smooth or PL. We study pairs $\left(J^{2 n+1}, K^{2 n-1}\right), n \geq 2$, with $J$ a sphere, and $K$ a homotopy sphere, smoothly or locally flat embedded in $J$. Such a knot admits a free $\mathbf{Z}_{m}$ action $T^{f}$, if $T^{f}$ acts freely on $J$, leaving $K$ invariant; it admits a semifree $\mathbf{Z}_{m}$ action $T^{s}$, if $T^{s}$ acts semifreely on $J$ with fixed set $J^{T}=K$. For a triple $(J, K ; T)$ with $T$ either free or semifree, an equivariant knot complement is a knot complement which is invariant under the action $T$. The algebraic invariants used are Seifert type invariants [L1], [L2], [St], [N]. We first prove:

THEOREM 2.3. A knot admits a free $\mathbf{Z}_{m}$ action if and only if it admits a semifree $\mathbf{Z}_{m}$ action. Moreover, the restrictions of these actions to equivariant knot complements can be chosen to be the same.

Two equivariant knots $\left(J_{i}, K_{i} ; T_{i}\right), i=0,1$, with $T_{i}$ both either free or semifree are equivariantly homeomorphic, if there exists a (PL, DIFF) homeomorphism $\phi: J_{0} \rightarrow J_{1}$ which is action and orientation preserving and such that $\phi\left(K_{0}\right)=K_{1}$.

THEOREM 2.4. Let $\left(J_{0}, K_{0}\right)$ and $\left(J_{1}, K_{1}\right)$ be two knots admitting $\mathbf{Z}_{m}$ actions. Then the fixed knots are equivariantly homeomorphic if 
and only if their associated action-equivalent invariant knots are equivariantly homeomorphic.

Here, two invariant knots $\left(\mathbf{Z}_{m}\right.$ acts freely) are action-equivalent, if their normal bundles are equivariantly isomorphic. For equivariant cobordism, we obtain:

THEOREM 2.5. A knot admitting $\mathbf{Z}_{m}$ actions is semifree nullcobordant if and only if it is free null-cobordant.

The precise relationship between the surgery theoretic CappellShaneson $\Gamma$ groups [CS1] in the cases of free and semifree actions will be given in a later paper. Here we obtain, as a result of Theorems 2.3, 2.4, and 2.5, the classification and the existence of fixed knots in terms of Seifert invariants, by using earlier results on invariant knots. In particular, we give the classification of simple fixed knots up to equivariant homeomorphism in terms of such invariants, by using our earlier classification [N] of invariant knots. (Recall that a knot is simple if its complement has the homotopy type of $S^{1}$ up to the middle dimension. Algebraic knots, for example, are always simple [M2].) Using $s$-equivalence of Seifert forms [L2] we prove:

THEOREM 3.2. Two simple fixed knots are equivariantly homeomorphic if and only if their derived Seifert forms are s-equivalent.

Here the derived Seifert form is the equivariant Seifert invariant defined in $\S 3$. A semifree action is highly connected if the fixed set is a simple knot. The corresponding classification of highly connected semifree actions on spheres is given in:

THEOREM 5.1. Two highly connected semifree actions on $S^{2 n+1}$, $n \geq 2$, are isomorphic if and only if their derived Seifert forms are s-equivalent.

Similarly, we use the equivariant cobordism classification of invariant knots [St], to give the corresponding classification for fixed knots in terms of cobordism of Seifert invariants:

THEOREM 3.3. A fixed knot is null-cobordant if and only if its derived Seifert matrix is null-cobordant. 
In $\S 3$, we use the realization of free actions on knots $[\mathbf{N}]$ to obtain necessary and sufficient conditions for the existence of semifree actions. This is done using the knot isometry $(Q, S)$ [M1], [St], [N]. Here $Q$ is the intersection form for a Seifert manifold $V$ of minimal genus, while $S$ is the rational monodromy of the knot. Recall that when these are given in matrix form and $B \in \mathscr{M}_{*}(\mathbf{Z})$ is a Seifert matrix for $V$, then $B=\left(I-S^{T}\right)^{-1} Q$, and the isometry $(Q, S)$ determines the knot.

THEOREM 3.6. Let $(J, K)$ be a simple knot with isometry $\left(Q, S_{0}\right)$ for the Seifert manifold $V$. Then $(J, K)$ admits a semifree $\mathbf{Z}_{m}$ action with equivariant Seifert manifold $V$, if and only if there exists a rational matrix $S_{1} \in \mathscr{M}_{*}(\mathbf{Q})$ such that:

1. $S_{1}^{m}=S_{0}$

2. $S_{1}^{T} Q S_{1}=Q$

3. $\left(I-S_{1}\right)^{-1} \in \mathscr{M}_{*}(\mathbf{Z})$.

In particular, for the unknot, we obtain:

COROLlary 3.7. The trivial knot admits a unique semifree $\mathbf{Z}_{m}$ action for each $m$.

Finally, we use Theorem 3.6 to improve on our calculations in [N].

TheOREM 4.1. The generalized trefoil knot, Seifert matrix:

$$
\left(\begin{array}{ll}
1 & 1 \\
0 & 1
\end{array}\right)
$$

is the unique genus 1 knot admitting semifree $\mathbf{Z}_{m}$ actions. This occurs for every $m \equiv \pm 1(\bmod 6)$, and the derived knot is also the generalized trefoil knot.

In $\S 6$ we use the results in $\S 2$ to study free $Z_{m}$ actions on spherical codimension two links. The results hold in high dimensions only; they are false in the classical dimension. We prove:

Corollary 6.2. Suppose $\mathbf{Z}_{m}$ acts freely on a codimension two spherical link. Then at most one component is invariant under this action, the other components being permuted.

We then use Corollary 6.2 to give necessary conditions for a link with four components to admit a free $\mathbf{Z}_{m}$ action, and then obtain 
a collection of four component links which cannot admit any such actions.

We note that C. Kearton, P. Strickland, and S. Wilson $[\mathbf{K}],[\mathbf{K W}]$, and [S], have solved the existence and equivariant homeomorphism problems of simple fixed knots in terms of the Blanchfield pairing. Hence Theorems 2.3 and 2.4 can be used also to give such results for invariant knots, in terms of the Blanchfield pairing.

I wish to thank Julius Shaneson and Mark Steinberger for helpful conversations, and Andrew Casson and Jacob Sturm for help with the calculations in $\S 4$.

1. Definitions and the derived knot. A fixed knot is a triple $\left(J^{2 n+1}, K^{2 n-1} ; T\right), n \geq 2$, where $J$ is a sphere, $K$ a homotopy sphere, and $T$ a semifree $\mathbf{Z}_{m}$ action on $J$ with fixed set $J^{T}=K$. Two such triples $\left(J_{0}, K_{0} ; T_{0}\right)$ and $\left(J_{1}, K_{1} ; T_{1}\right)$ are said to be equivariantly homeomorphic if there exists a (PL, DIFF) homeomorphism $\phi: J_{0} \rightarrow J_{1}$ which is action and orientation preserving, and such that $\phi\left(K_{0}\right)=\left(K_{1}\right)$. Note however that since the fixed set $K=J^{T}$ is determined by the action $T$, the condition $\phi\left(K_{0}\right)=\left(K_{1}\right)$ is unnecessary. Hence two fixed knots are equivariantly homeomorphic if and only if the corresponding actions are isomorphic. Two fixed knots $\left(J, K_{0} ; T_{0}\right)$ and $\left(J, K_{1} ; T_{1}\right)$ are equivariantly cobordant if there exists an $h$-cobordism $\mathscr{K}$ connecting $K_{0}$ and $K_{1}$ in $J \times I$, and a semifree $\mathbf{Z}_{m}$ action $\mathscr{T}$ on $J \times I$ with fixed set $\mathscr{K}$, and such that $\left.\mathscr{T}\right|_{J \times\{i\}}=T_{i}, i=0,1$.

Given a fixed knot $(J, K ; T)$, we consider a $T$-equivariant complement $X$, together with its orbit space $X^{*}=X / T$.

TheOREM 1.1. Let $(J, K ; T)$ be a fixed knot with knot group $\mathbf{Z}$, and $T$-equivariant complement $X$. Then the orbit space $X^{*}$ is also a knot complement. Moreover, $X$ and $X^{*}$ have the same homotopy groups.

Proof. Note that $X^{*}$ is a homology $S^{1}$. For the bundle map $\partial X \rightarrow K$ is a $T$-bundle with fiber $S^{1}$, and since $K$ is fixed, this fiber is a $T$-invariant meridian $\mu$. Hence if $p^{\prime}: \mu \rightarrow S^{1}$ is the restriction to $\mu$ of the $m$-fold cover $p: X \rightarrow X^{*}$, then the inclusion $\mu \hookrightarrow X$ induces an isomorphism of the spectral sequences of the two $m$-fold covers $p$ and $p^{\prime}$. Hence $X^{*}$ is a homology $S^{1}$. Consideration of the 
commutative diagram:

$$
\begin{aligned}
& 1 \rightarrow \pi_{1}(X) \stackrel{p}{\rightarrow} \pi_{1}\left(X^{*}\right) \stackrel{\phi}{\rightarrow} \mathbf{Z}_{m} \rightarrow 1 \\
& \cong \downarrow \quad \downarrow \\
& 1 \rightarrow H_{1}(X) \stackrel{p_{*}}{\rightarrow} H_{1}\left(X^{*}\right) \stackrel{\psi}{\rightarrow} \mathrm{Z}_{m} \rightarrow 1
\end{aligned}
$$

shows that $\pi_{1}\left(X^{*}\right)$ is abelian, hence isomorphic to $\mathbf{Z}$ (see [N], Proposition 2.1 for details). Since $p: X \rightarrow X^{*}$ is an $m$-fold cover, $\pi_{i}(X) \cong$ $\pi_{i}\left(X^{*}\right)$ for $i>1$. Notice too that since $\partial X \approx \mu \times K$ with $\mu$ a $T$-invariant circle, $\partial X / T=\partial X^{*} \approx S^{1} \times S^{2 n-1}$. Form the space $\mathscr{T}=X^{*} \cup_{\partial}\left(D^{2} \times S^{2 n-1}\right)$. Since $X^{*}$ is a homology $S^{1}$, it follows from Mayer-Vietoris that $\mathscr{T}$ is a homology $S^{2 n+1}$. Moreover, $\pi_{1}(\mathscr{T})=0$ by Van Kampen, since the map $j: \pi_{1}\left(\partial X^{*}\right) \rightarrow \pi_{1}\left(X^{*}\right)$ induced by inclusion is an isomorphism (see [N], 2.2). In DIFF the homotopy sphere $\mathscr{T}$ becomes a sphere by changing the smooth structure near a point outside $X^{*}$.

In $\mathscr{T}=X^{*} \cup\left(D^{2} \times S^{2 n-1}\right)$ we consider the codimension two subsphere $K^{\prime}=\{0\} \times S^{2 n-1}$. We call $\left(\mathscr{T}, K^{\prime}\right)$ the derived knot of the fixed knot $(J, K ; T)$. It follows from [L2] and the last statement of Theorem 1.1 that if $(J, K)$ is a simple knot, then the derived knot is determined by $X^{*}$. In general however, the derived knot as it is constructed here will be a pair of knots [LS], [CS2].

REMARK. We could, alternatively, construct the derived knot of a fixed knot by considering the quotient $S / T=\mathscr{T}$. If so, the derived knot will be unique even if $(J, K)$ is not simple. We prefer our method of definition, as it emphasizes the strong relationship between free and semifree actions.

2. Invariant knots versus fixed knots. We now use the derived knot construction to show that the cases of free and semifree $\mathbf{Z}_{m}$ actions on knots are essentially the same.

\section{Existence of Actions:}

Proposition 2.1. Let $\left(J, K ; T^{s}\right)$ be a fixed knot with $T^{s}$-equivariant complement $X$. Then $(J, K)$ admits a free $\mathbf{Z}_{m}$-action $T^{f}$ with equivariant complement $X$, such that $\left.T^{s}\right|_{X}=\left.T^{f}\right|_{X}$.

REMARK. The construction is similar to that in [St], except that in our case we obtain an arbitrary invariant knot, not necessarily simple. 
Proof. Choose a free $\mathbf{Z}_{m}$ action on $K$, and let $P$ denote the orbit space. Let $\xi$ be an oriented 2-plane bundle on $P$ with Euler class a given unit in $H^{2}(P ; \mathbf{Z})$. Denote by $(D(\xi), S(\xi))$ the associated $\left(D^{2}, S^{1}\right)$ bundle pair. It follows from the Gysin sequence that $S(\xi)$ is a homology $S^{1} \times S^{2 n-1}$, and from the homotopy sequence, a homotopy $S^{1} \times S^{2 n-1}$. Hence in PL, $S(\xi)=S^{1} \times S^{2 n-1}$ [B]. Form the space $Q=$ $D(\xi) \cup_{\partial} X^{*}$, where $X^{*}=X / T^{s}$ is the derived knot complement for the given fixed knot $\left(J, K ; T^{s}\right)$. Since $\left(X^{*}, \partial X^{*}\right)$ is a knot complement, it follows from Van Kampen that $\pi_{1}(Q) \cong \mathbf{Z}_{m}$, and its universal $(m$ fold) cover is the sphere $J^{\prime}=\left(K \times D^{2}\right) \cup_{\partial} X$. In DIFF the smooth structure of $Q$ may need to be modified in $D(\xi)$, i.e. away from $X^{*}$, in order to insure that the universal cover $J^{\prime}$ is a sphere. The $m$-fold cover $J^{\prime} \rightarrow Q$ provides a free $\mathbf{Z}_{m}$ action $T^{f}$ on the knot $\left(J^{\prime}, K\right)$, with equivariant complement $X$, and with $\left.T^{s}\right|_{X}=\left.T^{f}\right|_{X}$. If $(J, K)$ is simple, then it must be isotopic to $\left(J^{\prime}, K\right)$ since both knots have complement $X$. In the non-simple case, a twisting corresponding to the generator in $\pi_{1}(\mathrm{SO}(2 n))$ may occur. If so, we can modify the construction of $Q$ by attaching $(D(\xi), S(\xi))$ to $\left(X^{*}, \partial X^{*}\right)$ with a twist on the boundary.

Proposition 2.2. Let $\left(J, K ; T^{f}\right)$ be an invariant knot with $T^{f}$ equivariant complement $X$. Then $(J, K)$ admits a semifree $\mathbf{Z}_{m}$ action $T^{s}$, with equivariant complement $X$, such that $\left.T^{s}\right|_{X}=\left.T^{f}\right|_{X}$.

Proof. Consider the quotient of the pair $(J, K)$ by the free action $T^{f},(Q, P)=\left(J / T^{f}, K / T^{f}\right)$. The complement pair $\left(X^{*}, \partial X^{*}\right)$ of a tubular neighborhood of $P$ in $Q$, is the derived knot complement for the case of free cyclic actions [N]. Hence we have an $m$-fold cyclic cover

$$
\phi:(X, \partial X) \rightarrow\left(X^{*}, \partial X^{*}\right)
$$

with $(X, \partial X)$ a $T^{f}$-equivariant knot complement, and:

$$
\begin{aligned}
\partial X & =S^{1} \times K, \\
\partial X^{*} & =S^{1} \times_{T^{f}} K \approx S^{1} \times K .
\end{aligned}
$$

It therefore suffices to find a new trivialization of $\partial X$ such that $\partial X=$ $S^{1} / T^{f} \times K$. For then the action $\left.T^{f}\right|_{X}$ can be extended radially onto $D^{2} \times K$, giving the desired semifree action $T^{s}$. Consideration of the $m$-fold covers:

$$
\phi: \partial X \rightarrow \partial X^{*} \approx S^{1} \times K
$$


and, for (any) free $\mathbf{Z}_{m}$ action $\tau$ on $S^{1}$

$$
\psi: S^{1} \times K \rightarrow S^{1} / \tau \times K \approx \partial X^{*}
$$

provides an isomorphism between these two covers, and hence the desired new trivialization of $\partial X$.

It is worth stating Propositions 2.1 and 2.2 in:

THEOREM 2.3. A knot admits a free $\mathbf{Z}_{m}$ action if and only if it admits a semifree $\mathbf{Z}_{m}$ action. Moreover, the restrictions of these actions to equivariant knot complements can be chosen to be the same.

Equivariant classification. It is worth remarking on the degrees of freedom for constructing such actions. When starting with a semifree $\mathbf{Z}_{m}$ action on the knot $(J, K)$ any free action $\tau$ on the subsphere $K$, and any oriented 2-plane bundle on the orbit space $K / \tau$ with Euler class a unit, gives rise to a free action on $(J, K)$. We will soon see, however, that when starting with a free action on a knot, the associated semifree action on this knot does not depend on the equivariant normal bundle information.

For the case of knots invariant under a free cyclic action, we define two triples $\left(J_{0}, K_{0} ; T_{0}\right)$ and $\left(J_{1}, K_{1} ; T_{1}\right)$ to be action equivalent if their normal bundles $\nu\left(K_{i}, J_{i}\right)$ are equivariantly isomorphic. Comparing the equivariant classifications for the free and semifree case, we prove the following:

TheOREM 2.4. Let $\left(J_{0}, K_{0}\right)$ and $\left(J_{1}, K_{1}\right)$ be two knots admitting $\mathbf{Z}_{m}$ actions. Then the fixed knots are equivariantly homeomorphic if and only if their associated action-equivalent invariant knots are equivariantly homeomorphic.

Proof. For $j=0,1$ let $T_{j}^{s}$ be the semifree action, $T_{j}^{f}$ the free action, $X_{j}$ the knot complement, and $X_{j}^{*}$ the derived knot complement. (Recall $\left.\left.T^{s}\right|_{X}=\left.T^{f}\right|_{X}.\right)$ Suppose the fixed knots $\left(J_{0}, K_{0} ; T_{0}^{s}\right)$ and $\left(J_{1}, K_{1} ; T_{1}^{s}\right)$ are equivariantly homeomorphic via a map $\phi: J_{0} \rightarrow J_{1}$. Then

$$
\psi=\left.\phi\right|_{X_{0}}:\left(X_{0}, \partial X_{0}\right) \rightarrow\left(X_{1}, \partial X_{1}\right)
$$

extends to the normal bundles of the invariant knots, if and only if these bundles are $T^{f}$-equivariantly isomorphic [N]. Note that a twisting $\tau$ corresponding to the generator of $\pi_{1}(\mathrm{SO}(2 n))$ will not occur, since $\psi$ is the restriction of an isotopy. Conversely, suppose the invariant knots $\left(J_{0}, K_{0} ; T_{0}^{f}\right)$ and $\left(J_{1}, K_{1} ; T_{1}^{f}\right)$ are equivariantly 
homeomorphic via a map $\psi: J_{0} \rightarrow J_{1}$. Then $\phi=\left.\psi\right|_{\left(X_{0}, \partial X_{0}\right)}$ extends to the normal bundles of the fixed knots, if and only if these bundles are $T^{s}$ equivariantly isomorphic, since again the twisting $\tau$ cannot occur. But the fixed knot normal bundles will be equivariantly isomorphic if and only if the normal bundles of the derived knots are isomorphic. Since the derived knots are codimension 2 spherical knots, their normal bundles will always be trivial, hence isomorphic to each other.

For equivariant cobordism, let $(J, K)$ be a knot admitting a free $\mathbf{Z}_{m}$ action $T^{f}$ and associated semifree action $T^{s}$; denote by $T$ the common restriction of these actions to an equivariant knot complement. We say $(J, K ; T)$ is free null-cobordant if $\left(J, K ; T^{f}\right)$ is null-cobordant and semifree null-cobordant if $\left(J, K ; T^{s}\right)$ is nullcobordant.

THEOREM 2.5. A knot admitting $\mathbf{Z}_{m}$ actions is free null-cobordant if and only if it is semifree null-cobordant.

Proof. It follows from the definition of the derived knot for free actions, that an invariant knot is free null-cobordant if and only if the derived knot complement $X^{*}$ is cobordant rel boundary to the trivial knot complement; i.e. if and only if the derived knot is nullcobordant. For the semifree case let $\left(J, K ; T^{s}\right)$ be a fixed knot with $T^{s}$-equivariant complement $X$, derived knot $(\mathscr{T}, \Sigma)$, and derived knot complement $X^{*}$. Then an equivariant null-cobordism of $X$ is the same as a null-cobordism of $X^{*}$. The theorem now follows from the fact that the derived knot complements are the same in the free and in the semifree case.

3. Classification and realization of fixed knots. We now give the classification and realization theorems for fixed knots in terms of Seifert type invariants. An equivariant Seifert surface for a fixed knot $(J, K ; T)$ is a Seifert surface $V$ which is disjoint from its translates except on the boundary:

$$
V \cap T^{i}(V)=\partial V, \quad i=1, \ldots, m-1 .
$$

Proposition 3.1. A fixed knot has equivariant Seifert surfaces.

REMARKS.

- We work with the truncated Seifert surface, i.e. the intersection of the Seifert surface with a closed knot complement. 
- We could prove the result by lifting a Seifert surface for the derived knot to the $m$-fold cover. However, for reasons which will soon become clear, we use the associated invariant knot instead.

Proof. Let $\left(J, K ; T^{s}\right)$ be a fixed knot with associated invariant knot $\left(J, K ; T^{f}\right)$, and knot complement $X$. Let $V$ be a truncated equivariant Seifert surface for $\left(J, K ; T^{f}\right)$. (This always exists [St].) Then, since $\left.T^{s}\right|_{X}=\left.T^{f}\right|_{X}, V$ is also a truncated equivariant Seifert surface for $\left(J, K ; T^{s}\right)$. Extending $V$ radially throughout the fixed knot normal bundle gives the result.

REMARK. The proof of Proposition 3.1 shows that all algebraic invariants defined for invariant knots on truncated Seifert surfaces can also be defined for fixed knots. Moreover, these will have the same properties in both cases.

Let $\left(J^{2 n+1}, K^{2 n-1} ; T^{s}\right)$ be a fixed knot with derived knot $(\mathscr{T}, \Sigma)$, equivariant Seifert surface $V^{2 n}$, and derived Seifert surface $V^{* 2 n}$. A derived Seifert form will be the Seifert linking $\mathscr{B}$ for the derived knot $(\mathscr{T}, \Sigma)$ with Seifert surface $V^{*}$ (compare [N] for invariant knots).

$$
\begin{gathered}
\mathscr{B}: H_{n}\left(V^{*}\right) \otimes H_{n}\left(V^{*}\right) \rightarrow \mathbf{Z}, \\
\mathscr{B}(x, y)=L\left(x, \nu_{+} y\right) .
\end{gathered}
$$

Here $L$ is the linking in the derived knot complement, and $\nu_{+}$is a small push in the positive normal direction. Note that, since $V$ and $V^{*}$ are homeomorphic, $\mathscr{B}$ is defined on $H_{n}(V)$. It follows from our construction and the definition of linking numbers that

$$
\mathscr{B}(x, y)=\sum B_{i}(x, y)
$$

where $B_{i}(x, y)=L\left(x, T^{i}\left(\nu_{+} y\right), i=0, \ldots, m-1\right.$ [St], [N].

We now show that the notion of $s$-equivalence of Seifert matrices [L2] suffices for the classification of simple fixed knots up to equivariant homeomorphism.

THEOREM 3.2 (Equivariant Homeomorphism). Two simple fixed knots are equivariantly homeomorphic if and only if their derived Seifert forms are s-equivalent.

Proof. Notice that if $\left(J, K ; T^{s}\right)$ is a fixed knot with associated invariant knot $\left(J, K ; T^{f}\right)$, the derived Seifert forms for these two 
triples are the same. (Compare above with [N].) The result now follows from Theorem 2.4 together with the classification theorem for simple invariant knots [N].

Similarly, for equivariant cobordism of fixed knots we have:

Theorem 3.3 (Equivariant Cobordism). A fixed knot is null-cobordant if and only if its derived Seifert matrix is null-cobordant.

Proof. This follows from Theorem 2.5 and [St].

The question of existence of semifree actions on a given simple knot, is answered by using the following invariants determined by the Seifert forms (compare [St] for free actions). Given a fixed knot $\left(J, K ; T^{s}\right)$ with Seifert form $B$ and derived Seifert form $\mathscr{B}$ for the Seifert manifold $V$, define the isometric structure $\left(Q ; s_{0}, s_{1}\right)$ by:

(1) $Q=$ intersection form on $V,\left(Q=B \pm B^{T}\right)$,

(2) $s_{i} \in \operatorname{End}_{\mathbf{Z}}\left(H_{n}(V)\right) i=1,2$ defined by:

$$
\begin{aligned}
B(x, y) & =Q\left(s_{0} x, y\right), \\
\mathscr{B}(x, y) & =Q\left(s_{1} x, y\right) .
\end{aligned}
$$

Note that since $Q$ is unimodular, $s_{i}$ is well defined. If $s_{0}$ and $s_{1}$ are injective, we can also define the knot isometry $\left(Q ; S_{0}, S_{1}\right)$ where

$$
S_{i}=I-s_{i}^{-1}, \quad i=1,2 .
$$

In order to avoid the ambiguities caused by working over $\mathbf{Q}$, we regard these invariants as matrices in $\mathscr{M}_{*}(\mathbf{Q})$. If so, $Q=B \pm B^{T} \in \mathscr{M}_{*}(\mathbf{Z})$, $s_{0}=\left(Q^{T}\right)^{-1} B^{T}, s_{1}=\left(Q^{T}\right)^{-1} \mathscr{B}^{T}$, and $S_{i}=I-s_{i}^{-1} \in \mathscr{M}_{*}(\mathbf{Q})$. Notice then that

$$
B=\left(I-S_{0}^{T}\right)^{-1} Q, \quad \mathscr{B}=\left(I-S_{1}^{T}\right)^{-1} Q
$$

so that, as matrices, $\left(Q ; S_{0}\right)$ determines the original knot $(J, K)$, and $\left(Q, S_{1}\right)$ determines the derived knot.

Proposition 3.4. Whenever it is defined, the isometry $\left(Q ; S_{0}, S_{1}\right)$ for a fixed knot satisfies properties:

(i) $S_{1}^{m}=S_{0}$,

(ii) $S_{1}^{T} Q S_{1}=Q$,

(iii) $\left(I-S_{1}\right)^{-1} \in \mathscr{M}_{*}(\mathbf{Z})$.

Proof. Properties (i) and (ii) were proved in [St] for knots invariant under free actions. The proof for fixed knots is identical, hence we omit it. Property (iii) follows from the definition. 
We now show that $\left(Q ; S_{0}, S_{1}\right)$ can always be defined for a simple fixed knot; equivalently the isometric structure $\left(Q ; s_{0}, s_{1}\right)$ can always be assumed to be injective. A Seifert surface for a simple knot is minimal if ambient surgery cannot reduce its genus. An equivariant Seifert surface for a simple fixed knot is equivariantly minimal if ambient surgery cannot reduce its genus without producing a non-equivariant Seifert surface. Let $\left(J, K ; T^{s}\right)$ be a simple fixed knot with isometric structure $\left(Q ; s_{0}, s_{1}\right)$ for the equivariant Seifert surface $V$.

Proposition 3.5. (a) $V$ is minimal if and only if $s_{0}$ is injective, and it is equivariantly minimal if and only if $s_{1}$ is injective.

(b) $V$ is minimal if and only if it is equivariantly minimal.

Proof. See [N] Theorem 3.1 for the case of simple invariant knots with free cyclic actions.

Hence given a simple fixed knot, the isometry $\left(Q ; S_{0}, S_{1}\right)$ can always be defined. Notice that, since the Seifert matrices $(B, \mathscr{B})$ can be recovered from the isometry, the isometry determines the fixed knot up to equivariant homeomorphism.

THEOREM 3.6. Let $(J, K)$ be a simple knot with isometry $\left(Q, S_{0}\right)$ for the Seifert manifold $V$. Then $(J, K)$ admits a semifree $\mathbf{Z}_{m}$ action with equivariant Seifert manifold $V$, if and only if there exists a rational matrix $S_{1} \in \mathscr{M}_{*}(\mathbf{Q})$ such that:

1. $S_{1}^{m}=S_{0}$,

2. $S_{1}^{T} Q S_{1}=Q$,

3. $\left(I-S_{1}\right)^{-1} \in \mathscr{M}_{*}(\mathbf{Z})$.

Proof. If an action exists, then $S_{1}$ exists by Proposition 3.5, and properties (i), (ii) and (iii) must be satisfied by Proposition 3.4. Suppose conversely that $S_{1}$ exists. By [St] and [N], $(J, K)$ admits a free $\mathbf{Z}_{m}$ action $T^{f}$ with isometry $\left(Q ; S_{0}, S_{1}\right)$. by Theorem 2.3 we can let $T^{s}$ be the associated semifree action on $(J, K)$, and its isometry will still be $\left(Q ; S_{0}, S_{1}\right)$. (See Remark after Proposition 3.1.)

It is worth mentioning the special case of the trivial knot:

COROLlaRY 3.7. The trivial knot admits a unique semifree $\mathbf{Z}_{m}$ action for each $m$.

Let $\phi(m)$ be the Euler function and $\Theta_{m}$ the isomorphism classes of free $\mathbf{Z}_{m}$ actions on $S^{2 n-1}$. 
COROllary 3.8. The trivial knot admits $\phi(m)\left|\Theta_{m}\right|$ different free $\mathbf{Z}_{m}$ actions for each $m$.

Proof. Let $(J, K ; T)$ be an invariant knot with quotient lens space pair $(Q, P)$. The action-equivalence class of $(J, K ; T)$ depends on (see construction in Proposition 2.1) the action of $T$ on $K$, together with a choice of Euler class for the normal bundle $\nu(P, Q)$. The result now follows from the observation that each generator in $H^{2}(P ; \mathbf{Z}) \cong$ $\mathbf{Z}_{m}$ provides such an Euler class.

4. Calculations. Theorem 3.6 allows for concrete results through matrix computations. The results on free $\mathbf{Z}_{m}$ actions on fibered genus 1 knots carry over from [N], but we give here stronger results, solving the problem for knots not necessarily fibered. Notice that in the fibered case, condition 3 of Theorem 3.6 is equivalent to:

$$
\operatorname{det}\left(I-S_{1}\right)^{-1}= \pm 1
$$

since the knot fibers if and only if $S_{1} \in \mathscr{M}_{*}(\mathbf{Z})$ [N]. For the general genus 1 case, we use the weaker condition

$$
\operatorname{ch}_{\left(I-S_{j}\right)^{-1}}(t) \in \mathbf{Z}[t], \quad j=0,1,
$$

where $\operatorname{ch}_{\left(I-S_{J}\right)^{-1}}(t)$ is the characteristic polynomial.

Symmetric case. It is easy to see that no matrix $S \in \mathscr{M}_{*}(\mathbf{Q})$ satisfies conditions (2) and (3) of Theorem 3.6, so there is nothing to check.

Skew-symmetric case.

$$
Q=\left(\begin{array}{cc}
0 & 1 \\
-1 & 0
\end{array}\right)
$$

We find triples $\left(S_{0}, S_{1} ; m\right)$ such that:

1. $S_{1}^{m}=S_{0}$,

2. $S_{i} \in \mathrm{SL}_{2}(\mathbf{Q})$,

3. $\left(I-S_{i}\right)^{-1} \in \mathscr{M}_{2}(\mathbf{Z})$.

Note that for $Q$ as above $S_{i} \in \mathrm{SL}_{2}(\mathbf{Q})$ if and only if $S_{i}$ is an isometry for $Q$. Suppose $\left(S_{0}, S_{1} ; m\right)$ is such a triple.

Claim 1. $S_{1}$ is diagonalizable over $\mathbf{C}$.

Proof. Suppose

$$
S_{1} \sim\left(\begin{array}{cc}
\varepsilon & A \\
0 & \varepsilon
\end{array}\right)
$$


where " $\sim$ " denotes similarity over $\mathbf{C}$ and, since $\operatorname{det} S_{1}=1, \varepsilon=$ \pm 1 . But if $\varepsilon=1$, then $\left(I-S_{1}\right)$ is singular, and if $\varepsilon=-1$, then $\operatorname{ch}_{\left(I-S_{1}\right)^{-1}}(t)=(t-1 / 2)^{2} \notin \mathbf{Z}[t]$.

Letting $\lambda_{1}, \lambda_{2}$ be the eigenvalues for $S_{1}$, we conclude from $\left(3^{\prime \prime}\right)$ that:

$$
\begin{gathered}
\left(\left(\begin{array}{ll}
1 & 0 \\
0 & 1
\end{array}\right)-\left(\begin{array}{cc}
\lambda_{1} & 0 \\
0 & \lambda_{2}
\end{array}\right)\right)^{-1}=\left(\begin{array}{cc}
a_{1} & 0 \\
0 & a_{2}
\end{array}\right) \\
\left(\left(\begin{array}{ll}
1 & 0 \\
0 & 1
\end{array}\right)-\left(\begin{array}{cc}
\lambda_{1}^{m} & 0 \\
0 & \lambda_{2}^{m}
\end{array}\right)\right)^{-1}=\left(\begin{array}{cc}
b_{1} & 0 \\
0 & b_{2}
\end{array}\right),
\end{gathered}
$$

where $a_{1}, a_{2}, b_{1}$, and $b_{2}$ are algebraic integers.

Letting $k=\mathbf{Q}\left(a_{1}, a_{2}\right)$, we have $[k: \mathbf{Q}]=1$ or 2 . Let $\mathscr{O}_{k}$ denote the ring of integers in $k$ and $\mathscr{O}_{k}^{*}$ the units.

Claim 2. $a_{j}^{m}-\left(a_{j}-1\right)^{m} \in \mathscr{O}_{k}^{*}$.

Proof. Note that $b_{j}=a_{j}^{m}\left(a_{j}^{m}-\left(a_{j}-1\right)^{m}\right)^{-1}$. Since $b_{j}$ is an algebraic integer, we get $\left(a_{j}^{m}-\left(a_{j}-1\right)^{m}\right)$ divides $a_{j}^{m}$. But $a_{1}+a_{2}=1$, and hence $\left(a_{j}^{m}-\left(a_{j}-1\right)^{m}\right)$ also divides $\left(a_{j}-1\right)^{m}$. Since $a_{j}$ and $\left(a_{j}-1\right)$ are relatively prime, the claim follows.

Claim 3. $[k: \mathbf{Q}] \neq 1$.

Proof. Otherwise $a_{j} \in \mathbf{Z}$ and $a_{j}^{m}-\left(a_{j}-1\right)^{m}= \pm 1$. Let $m=l p$, $p$ any prime. Then

$$
\pm 1=\left[a_{j}^{p}-\left(a_{j}-1\right)^{p}\right]\left[a_{j}^{l-1}+\cdots+\left(a_{j}-1\right)^{l-1}\right] .
$$

Both factors are integers and units, so that

$$
\pm 1=a_{j}^{p}-\left(a_{j}-1\right)^{p}
$$

If $p=2$, then $a_{j}=0$ or 1 . Since $a_{1}+a_{2}=1,\left(I-s_{1}\right)^{-1}$ is singular, which is impossible. If $p$ is an odd prime, then

$$
\pm 1=a_{j}^{p}-\left(a_{j}-1\right)^{p} \equiv 1 \quad(\bmod p) .
$$

Hence

$$
1=a_{j}^{p}-\left(a_{j}-1\right)^{p}=p a_{j}^{p-1}-\left(\begin{array}{c}
p \\
2
\end{array}\right) a_{j}^{p-2}+\cdots-p a_{j}+1 .
$$

Hence

$$
a_{j}\left(a_{j}^{p-3}-\frac{1}{p}\left(\begin{array}{l}
p \\
2
\end{array}\right) a_{j}^{p-4}+\cdots+1\right)=1 .
$$


Since $p$ is prime, both factors are integers and units. In particular $a_{j}= \pm 1$, which is impossible, since $a_{1}+a_{2}=1$.

Hence $[k: \mathbf{Q}]=2$.

Claim 4. $\lambda_{j}=e^{ \pm \pi i / 3}$, and $m \equiv \pm 1(\bmod 6)$.

Proof. Let $\sigma$ be the non-trivial element in $\operatorname{Gal}(k / \mathbf{Q})$. Let $m=l p$ for any prime $p$. Since

$$
\left(a_{j}^{p}-\left(a_{j}-1\right)^{p}\right)[\text { algebraic integer }]=a_{j}^{m}-\left(a_{j}-1\right)^{m} \in \mathscr{O}_{k}^{*}
$$

we again conclude that $\eta_{j}=a_{j}^{p}-\left(a_{j}-1\right)^{p} \in \mathscr{O}_{k}^{*}$. Note that $\sigma\left(\eta_{1}\right)=\eta_{2}$, hence $\eta_{1} \eta_{2}= \pm 1$.

If $p=2$, then $\eta_{1}=-\eta_{2}$ so $\eta_{j}= \pm 1$ or $\eta_{j}= \pm i$. But if $\eta_{j}= \pm 1$, then $a_{j} \in \mathbf{Q}$ which is impossible, while if $\eta_{j}= \pm i$ then $a_{j}=1 / 2(1 \pm i)$ which is not an algebraic integer.

If $p$ is odd, then $\eta_{1}=\eta_{2}$ so $\sigma$ fixes $\eta_{j}$. Hence $\eta_{j}= \pm 1$. As in Claim 3 , since $p$ is an odd prime, $\eta_{j}=1$, so

$$
a_{j} \text { [algebraic integer] }=1 \text {. }
$$

Thus $a_{j} \in \mathscr{O}_{k}^{*}$ and $a_{1} a_{2}=a_{1} \sigma\left(a_{1}\right)= \pm 1$. Since $a_{1}+a_{2}=1$, the minimum polynomial for $a_{j}$ is:

$$
\mu(t)=t^{2}-t+1 \text { or } \nu(t)=t^{2}-t-1 .
$$

In particular, either

$$
a_{j}=e^{ \pm \pi i / 3} \quad \text { or } \quad a_{j}=\frac{1 \pm \sqrt{5}}{2}
$$

We now consider the Alexander polynomials of these knots to show that $a_{j}=(1 / 2)(1 \pm \sqrt{5})$ cannot occur, while $a_{j}=e^{ \pm \pi i / 3}$ occurs only when $m \equiv \pm 1(\bmod 6)$. Let $\operatorname{ch}_{j}(t), j=0,1$, be the characteristic polynomial for $S_{j}$, and $\Delta_{j}(t)$ the Alexander polynomial of the knot $\left(Q, S_{j}\right)$ with Seifert matrix $B^{j}$. (Note that $B^{0}=B$ the Seifert matrix for the original fixed knot, and $B^{1}=\mathscr{B}$ the derived Seifert matrix.) Recall that $\Delta_{j}(t)=\operatorname{det}\left(B^{j}\right) \operatorname{ch}_{j}(t)$ and that $\Delta_{j}(1)= \pm 1$. If $\operatorname{ch}_{1}(t)=$ $t^{2}-t-1$ then $\operatorname{ch}_{1}(1)=-1$, so $\operatorname{det}\left(B^{1}\right)= \pm 1$. Hence the derived knot is fibered, and hence so must the original (fixed) knot be. Similarly, if $\operatorname{ch}_{1}(t)=t^{2}-t+1$ both the derived and the original (fixed) knot must be fibered. We could now invoke the results in [N] on fibered genus 1 knots, but we need not do so. For, since the knots are fibered,

$$
\pm \Delta_{0}(t)=\operatorname{ch}_{0}(t)=t^{2}-t\left(\lambda_{1}^{m}+\lambda_{2}^{m}\right)+1 \text {. }
$$


But $\operatorname{ch}_{0}(1)= \pm 1$ if and only if $f(m)=\lambda_{1}^{m}+\lambda_{2}^{m}=1$ or 3 . If $a_{j}=(1 / 2)(1 \pm \sqrt{5})$, then $f(m)$ is increasing, with $f(1)=3$. Hence only the trivial group can act. If $a_{j}=e^{ \pm \pi i / 3}$ then

$$
f(m)=\left\{\begin{aligned}
1, & \text { if } m \equiv \pm 1(\bmod 6) \\
-1, & \text { if } m \equiv \pm 2(\bmod 6) \\
2, & \text { if } m \equiv 0(\bmod 3)
\end{aligned}\right.
$$

This finishes the proof of Claim 4 but we still must show that triples $\left(S_{0}, S_{1} ; m\right)$ with $\lambda_{j}=e^{ \pm \pi i / 3}$ and $m \equiv \pm 1(\bmod 6)$ do in fact occur. Note that, since

$$
S_{1} \sim\left(\begin{array}{cc}
e^{\pi i / 3} & 0 \\
0 & e^{-\pi i / 3}
\end{array}\right)
$$

$S_{1}$ is periodic with period 7 . Notice also that

$$
\left(\begin{array}{ll}
0 & 1 \\
1 & 0
\end{array}\right) S_{1}^{5}\left(\begin{array}{ll}
0 & 1 \\
1 & 0
\end{array}\right)^{-1}=S_{1} \text {. }
$$

Hence each $m \equiv \pm 1(\bmod 6), m>0$, will occur (with original and derived knot isotopic), as soon as $S_{1}$ with properties (2) and (3) is produced, such that

$$
S_{1} \sim\left(\begin{array}{cc}
e^{\pi i / 3} & 0 \\
0 & e^{-\pi i / 3}
\end{array}\right)
$$

We show that there exists a unique knot with the above properties. Since the knot must be fibered, we may assume $S_{1} \in \mathrm{SL}_{2}(\mathbf{Z})$.

Claim 5. The matrix

$$
\mathscr{L}=\left(\begin{array}{cc}
1 & -1 \\
1 & 0
\end{array}\right)
$$

satisfies the above mentioned properties, and every matrix $S_{1} \in \mathrm{SL}_{2}(\mathbf{Z})$ satisfying these properties is similar in $\mathrm{SL}_{2}(\mathbf{Z})$ to $\mathscr{L}$.

Proof. It is easy to check that $\mathscr{L}$ satisfies properties (2) and (3). Notice now that in $\operatorname{PSL}_{2}(\mathbf{Z})=\mathrm{SL}_{2}(\mathbf{Z}) /\{ \pm I\},\left[ \pm S_{1}\right]$ and $[ \pm \mathscr{L}]$ are similar. For the group of conjugacy classes in $\operatorname{PSL}_{2}(Z)$ is $\left\{\alpha, \beta \mid \alpha^{2}=\right.$ $\left.\beta^{3}=1\right\}$ with all elements of order 3 conjugate to each other. But $S_{1}^{3}=-I$. Hence $\left[ \pm S_{1}\right] \sim[ \pm \mathscr{L}]$ in $\operatorname{PSL}_{2}(Z)$. Equivalently $S_{1} \sim \mathscr{L}$ or $S_{1} \sim-\mathscr{L}$ in $\mathrm{SL}_{2}(\mathbf{Z})$. But $\operatorname{tr}\left(S_{1}\right)=1$ and $\operatorname{tr}(-\mathscr{L})=-1$, where tr denotes the trace. Hence $S_{1} \sim \mathscr{L}$ in $\mathrm{SL}_{2}(\mathbf{Z})$.

We summarize in the following: 
THEOREM 4.1. The generalized trefoil knot

$$
\text { Seifert matrix } B=\left(\begin{array}{ll}
1 & 1 \\
0 & 1
\end{array}\right)
$$

is the unique genus 1 knot admitting a semifree $\mathbf{Z}_{m}$ action. This occurs if and only if $m \equiv \pm 1(\bmod 6)$, and for each such $m$ the action is unique. Moreover, the derived knot is also the generalized trefoil knot.

If $\left(Q ; S_{0}, S_{1}\right)$ and $\left(Q^{\prime} ; S_{0}^{\prime}, S_{1}^{\prime}\right)$ are two simple $\mathbf{Z}_{m}$-fixed knots, we define their connected sum to be the triple $\left(Q \oplus Q^{\prime} ; S_{0} \oplus S_{0}^{\prime}, S_{1} \oplus S_{1}^{\prime}\right)$ where $\oplus$ denotes block sum. We define $n\left(Q ; S_{0}, S_{1}\right)$ to be the connected sum of $n$ copies of $\left(Q ; S_{0}, S_{1}\right)$. As in [N], we can construct two different $\mathbf{Z}_{5}$ actions on 4 (generalized trefoil knot)

$$
\left(Q ; S_{0}\right)=4\left(\left(\begin{array}{cc}
0 & 1 \\
-1 & 0
\end{array}\right) ;\left(\begin{array}{cc}
0 & -1 \\
1 & 1
\end{array}\right)\right)
$$

The first is the natural $\mathbf{Z}_{5}$ action

$$
\left(Q ; S_{0}, S_{1}\right)=4\left(\left(\begin{array}{cc}
0 & 1 \\
-1 & 0
\end{array}\right) ;\left(\begin{array}{cc}
0 & -1 \\
1 & 1
\end{array}\right),\left(\begin{array}{cc}
1 & 1 \\
-1 & 0
\end{array}\right)\right) \text {. }
$$

Here

$$
\left(\begin{array}{cc}
1 & 1 \\
-1 & 0
\end{array}\right)^{5}=\left(\begin{array}{cc}
0 & -1 \\
1 & 1
\end{array}\right)
$$

and

$$
\left(\begin{array}{cc}
0 & -1 \\
1 & 1
\end{array}\right) \sim\left(\begin{array}{cc}
1 & 1 \\
-1 & 0
\end{array}\right) \sim\left(\begin{array}{cc}
1 & -1 \\
1 & 0
\end{array}\right) \quad \text { in } \quad \operatorname{SL}_{2}(\mathbf{Z})
$$

where the third matrix is $\mathscr{L}$ of Claim 5.

The second $\mathbf{Z}_{5}$ action on 4 (generalized trefoil knot) is a fixed knot which does not decompose equivariantly into a connected sum. (See [N] for details in the case of invariant knots.)

We therefore conclude:

TheOREM 4.2. There exist knots admitting different semifree $\mathbf{Z}_{m}$ actions with non-isotopic derived knots. In particular, there exist connected sums of knots admitting actions making them equivariantly indecomposable.

5. Classifying semifree actions on odd dimensional spheres. We consider semifree $\mathbf{Z}_{m}$ actions on spheres with fixed set a codimension two knot. Given such an action $T$ on a sphere $J$, define the knot of the action to be $\left(J, J^{T}\right)$; a Seifert form will be the derived Seifert form for 
the fixed knot. Notice that two semifree actions are isomorphic if and only if their knots are equivariantly homeomorphic. A semifree action is highly connected if its knot is simple. It is worth reinterpreting some of the fixed knots results in $\S 3$ and $\S 4$ in terms of classification and existence results for semifree actions on spheres. Thus Theorem 3.2 becomes:

THEOREM 5.1. Two highly connected semifree actions on $S^{2 n+1}$, $n \geq 2$, are isomorphic if and only if their Seifert forms are s-equivalent.

Corollary 3.7 and the calculations in $\S 4$ can be summarized in:

Proposition 5.2. For each $m$ there exists a unique semifree $\mathbf{Z}_{m}$ action on $S^{2 n+1}$ with fixed set the unknot.

Proposition 5.3. $S^{4 n+1}$ admits no semifree $\mathbf{Z}_{m}$ actions with fixed set a genus one knot. $S^{4 n-1}$ admits a semifree $\mathbf{Z}_{m}$ action with fixed set a genus one knot, if and only if $m \equiv \pm 1$ (mod 6). Moreover, for each such $m$ the action is unique up to isomorphism.

6. Free $\mathbf{Z}_{m}$ actions on links. Unlike the semifree case, where an action $T$ on a sphere $J$ determines the fixed knot $\left(J, J^{T}\right)$, a free $\mathbf{Z}_{m}$ action $T$ on $J$ does not in general determine a unique invariant knot.

EXAMPLE. Let $\mathbf{Z}_{5}$ act freely on the unit sphere in $\mathbf{C}^{n+1}$ by:

$$
T\left(z_{1}, \ldots, z_{n}, z_{n+1}\right)=\left(\rho^{3} z_{1}, \ldots, \rho^{3} z_{n}, \rho^{2} z_{n+1}\right)
$$

where $\rho=e^{2 \pi i / 5}$. Then $T$ leaves the (trivial) knot $z_{n+1}=0$ invariant. $T$ also leaves invariant the link of the singularity at 0 for the polynomial equation:

$$
z_{1}^{2}+\cdots+z_{n}^{2}+z_{n+1}^{3}=0
$$

which, when $n$ is odd, is a spherical non-trivial knot [M2]. The latter is in fact the $Z_{5}$ action on the generalized trefoil knot found in $\S 4$. However, we can prove the following:

Proposition 6.1. If $T$ is a free $\mathbf{Z}_{m}$ action on a sphere $J$ then any two $T$-invariant codimension two knots in $J$ must intersect.

Proof. Notice first that $m$ may be assumed to be prime. For if $p$ is a prime dividing $m$, then the free $\mathbf{Z}_{m}$ action $T$ naturally restricts to a free $\mathbf{Z}_{p}$ action $T^{\prime}$ for which $K_{0}$ and $K_{1}$ are still invariant. 
Suppose $\left(J, K_{0} ; T^{f}\right)$ and $\left(J, K_{1} ; T^{f}\right)$ are such that $K_{0} \cap K_{1}=\varnothing$. Consider the associated fixed knot $\left(J, K_{0} ; T_{0}^{s}\right)$ where the action is modified in a normal bundle of $K_{0}$ disjoint from $K_{1}$. The invariant knot $K_{1}$ is then contained in the complement of this fixed knot. The same construction for $\left(J, K_{1} ; T^{f}\right)$ yields the fixed knot $\left(J, K_{1} ; T_{1}^{S}\right)$. By Theorem 2.3, each one of the two semifree actions $T_{0}^{s}$ and $T_{1}^{s}$ agrees with the original free action $T^{f}$ on an equivariant link complement $X$. Hence we can construct a semifree $\mathbf{Z}_{m}$ action:

$$
\tau= \begin{cases}T^{f} & \text { on } X, \\ T_{i}^{s} & \text { on } \nu\left(K_{i}\right), \quad i=0,1 .\end{cases}
$$

Hence $\tau$ is a (prime order) semifree $\mathbf{Z}_{m}$ action with fixed set a disjoint union of two spheres, which is impossible by Smith theory. (See also [C].)

Hence we conclude the following result for free actions on codimension 2 high dimensional spherical links:

COROLlaRY 6.2. Suppose $\mathbf{Z}_{m}$ acts freely on a codimension two spherical link. Then at most one component is invariant under this action, the other components being permuted.

Let $\mathscr{L}$ be a link. Its order $|\mathscr{L}|$ denotes the number of components of the link. An isotopy partition of $\mathscr{L}$ is a partition into sublinks $L_{1}, \ldots, L_{k}$ such that: (a) for each $i$ the components of $L_{i}$ are isotopic to each other, and (b) the components of $L_{i}$ are never isotopic to those of $L_{j}$ for $i \neq j$. We call the sublinks $L_{j}$ of such a partition, the isotopy sublinks.

Proposition 6.3. Let $\mathbf{Z}_{m}$ act freely on a link $\mathscr{L}$ with isotopy partition $L_{1}, \ldots, L_{k}$. Then either:

(a) $m|| L_{i} \mid$ for $i=1, \ldots, k$ or

(b) $\exists j$ such that $m|| L_{i} \mid$ for $i \neq j$, and $m|| L_{j} \mid-1$.

Proof. If $K$ is a component of $\mathscr{L}$ and $\mathscr{O}_{K}$ is its orbit under the action, then $\mathscr{O}_{K}$ is contained in an isotopy sublink. It now follows from Corollary 6.2 that, with the possible exception of a unique comporient $K$, every orbit $\mathscr{O}_{K}$ must have exactly $m$ components.

Suppose $T$ is a free $\mathbf{Z}_{m}$ action on a link $\mathscr{L}$ with $k$ components. Regard an element $t$ of $T$ as a permutation on $k$ letters ( $k$ link 
components), written as a product of disjoint cycles:

$$
t=\sigma_{1} \cdots \sigma_{n} .
$$

It follows from the proof of Proposition 6.3 that $\operatorname{order}\left(\sigma_{i}\right)=m$ for each $i$, and that $t$ fixes at most one letter (equivalently $t$ leaves at most one component invariant).

We study the case of links with four components $\mathscr{L}=\left(K_{1}, \ldots, K_{4}\right)$.

Caution. The sketches which follow are in the classical dimension and are included only for the sake of clarity. The results are in high dimensions.

Let $t$ be a generator of the free $\mathbf{Z}_{m}$ action $T$ on the four component link $\mathscr{L}$.

Case 1. $t$ fixes one letter.

Then $t=\sigma_{1} \cdots \sigma_{n}$ is a permutation on 3 letters, and since $\operatorname{order}\left(\sigma_{i}\right)$ $=m$ for each $i, t$ is a 3-cycle, $t=\left(K_{1}, K_{2}, K_{3}\right)$. Hence $m=3$, and the following hold:

(a) the components $K_{1}, K_{2}$, and $K_{3}$ are isotopic knots;

(b) the 2-component sublinks: $\left(K_{1}, K_{2}\right),\left(K_{2}, K_{3}\right)$, and $\left(K_{3}, K_{1}\right)$, have the same link type;

(c) the 2-component sublinks $\left(K_{4}, K_{i}\right), i=1,2,3$, have the same link type; and,

(d) the 3-component sublinks $\left(K_{4}, K_{i}, K_{j}\right), 1 \leq i<j \leq 3$, have the same link type.

An example of such a link in the classical dimension is:

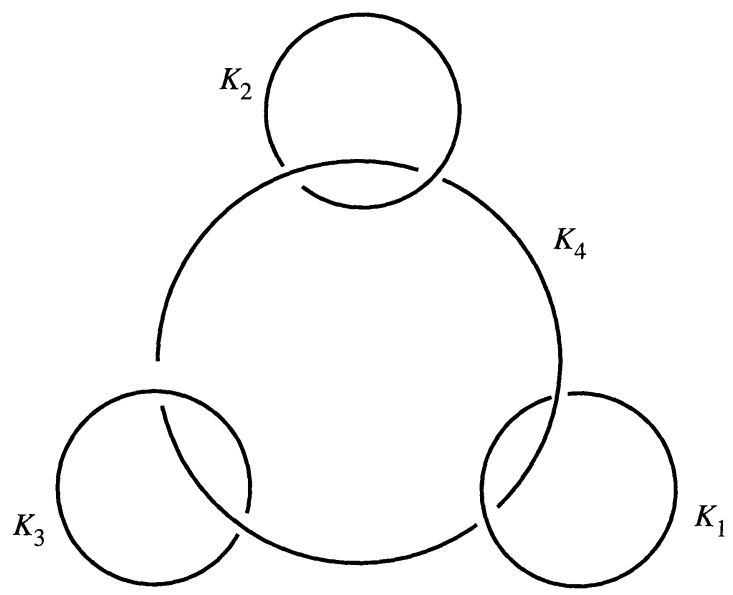


Case 2. $t$ fixes no letter.

Then $t$ is either (a) a product of two disjoint transpositions, hence $m=2$, or (b) a 4-cycle and $m=4$.

(a) $t=\left(K_{1}, K_{2}\right)\left(K_{3}, K_{4}\right)$ gives:

(i) $K_{1}$ and $K_{2}$ are isotopic,

$K_{3}$ and $K_{4}$ are isotopic

and

(ii) $\left(K_{1}, K_{3}\right)$ and $\left(K_{2}, K_{4}\right)$ are the same link type, $\left(K_{1}, K_{4}\right)$ and $\left(K_{2}, K_{3}\right)$ are the same link type.

In the classical dimension an example is:
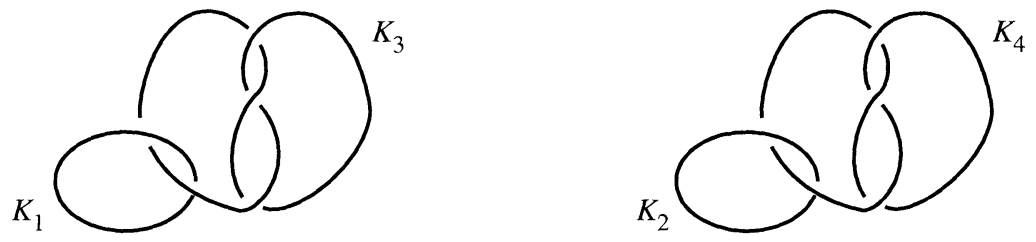

(b) $t=\left(K_{1}, K_{3}, K_{2}, K_{4}\right)$ :

(i) $K_{1}, K_{2}, K_{3}$, and $K_{4}$ are isotopic,

(ii) $\left(K_{1}, K_{3}\right),\left(K_{2}, K_{3}\right),\left(K_{2}, K_{4}\right)$ and $\left(K_{4}, K_{1}\right)$ are the same link type.

In the classical dimension an example is:

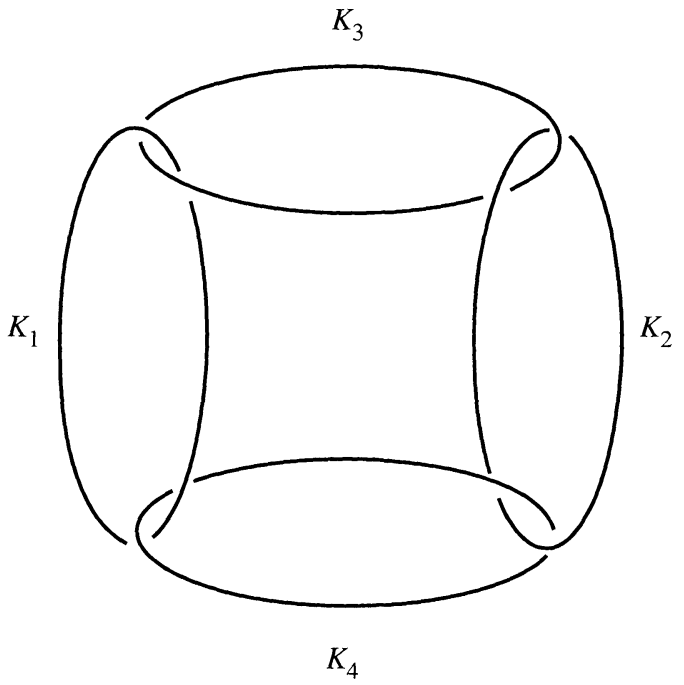


Let $\mathscr{L}=\left(K_{1}, K_{2}, K_{3}, K_{4}\right)$ be a link whose sublinks of order 2 are: $L_{1}, L_{2}, L_{1}^{\prime}, L_{2}^{\prime}, L_{3}^{\prime}$, and $L_{4}^{\prime}$. Suppose $L_{1}$ and $L_{2}$ have a component in common, and suppose $L_{i}$ and $L_{j}^{\prime}$ are never the same link type.

We then have:

Proposition 6.4. Such a link $\mathscr{L}$ with four components never admits free $\mathbf{Z}_{m}$ actions.

Proof. Clearly $\mathscr{L}$ does not satisfy the conditions in Case 1 or Case 2(a) or (b).

An example of such a link in the classical dimension is:
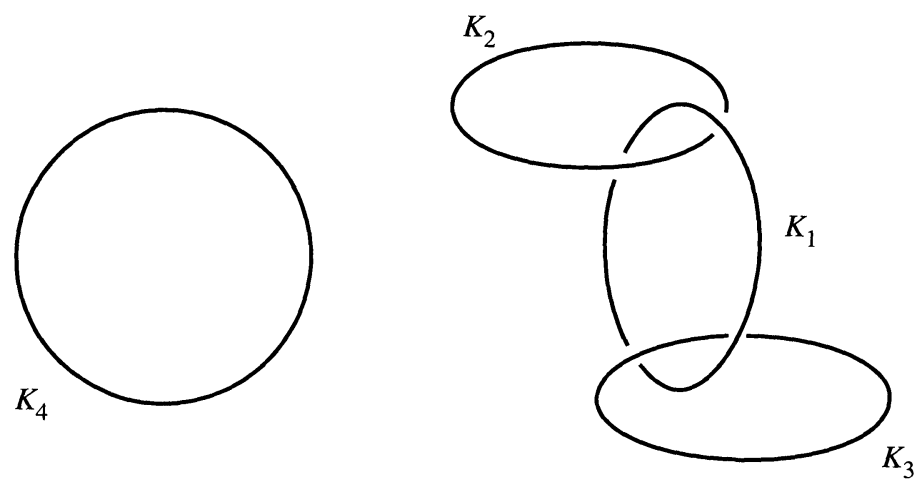

Here we take $L_{1}=\left(K_{1}, K_{2}\right)$ and $L_{2}=\left(K_{1}, K_{3}\right)$.

REMARK. If $\mathscr{L}$ is any link with isotopy sublinks $L_{1}, \ldots, L_{k}$ and such that the union of several isotopy sublinks is a link of order 4 of the type in Proposition 6.4, then $\mathscr{L}$ still will not admit any free $\mathbf{Z}_{m}$ actions.

\section{REFERENCES}

[B] W. Browder, Manifolds with $\pi_{1}=$ Z, Bull. Amer. Math. Soc., 64 (1966), 238-244.

[CS1] S. Cappell and J. L. Shaneson, The codimension two placement problem and homology equivalent manifolds, Ann. of Math., (2) 99 (1974), 277-348.

[CS2] __ There exist inequivalent knots with the same complement, Ann. of Math., (2) 103 (1976), 349-353.

[C] R. N. da Cruz, Periodic knots, Thesis, Courant Institute, 1987. 
[K] C. Kearton, Spinning, factorization of knots, and cyclic group actions on spheres, Arch. Math., 40 (1983), 361-363.

[KW] C. Kearton and S. M. J. Wilson, Cyclic group actions on odd-dimensional spheres, Comment. Math. Helv., 56 (1981), 615-626.

[LS] R. K. Lashof and J. L. Shaneson, Classification of knots in codimension two, Bull. Amer. Math. Soc., 75 (1969), 171-175.

[L1] J. Levine, Knot cobordism in codimension two, Comment. Math. Helv., 44 (1969), 229-244.

[L2] An algebraic classification of some knots of codimension two, Comment. Math. Helv., 45 (1970), 185-198.

[M1] J. Milnor, Infinite cyclic coverings, in Conference on the Topology of Manifolds, Prindle, Weber and Schmidt, Boston, pp. 115-133.

[M2] _ Singular Points of Complex Hypersurfaces, Princeton University Press, Princeton, NJ, 1968.

[N] M. Nicolau, A classification of invariant knots, Duke Math. J., (1) 58 (1989), 151-171.

[St] N. Stoltzfus, Equivariant concordance of invariant knots, Trans. Amer. Math. Soc., 254 (1979), 1-45.

[S] P. Strickland, Branched cyclic covers of simple knots, Proc. Amer. Math. Soc., (3) 90 (1984), 440-444.

Received December 18, 1990. Partially supported by NSF grant DMS 84-21371.

UNIVERSITY OF ILLINOIS

URBANA, IL 61801 


\title{
PACIFIC JOURNAL OF MATHEMATICS \\ Founded by \\ E. F. BeCKenbach (1906-1982) F. Wolf (1904-1989)
}

\section{EDITORS}

\author{
V. S. VARADARAJAN \\ (Managing Editor) \\ University of California \\ Los Angeles, CA 90024-1555 \\ vsv@math.ucla.edu \\ Herbert Clemens \\ University of Utah \\ Salt Lake City, UT 84112 \\ clemens@math.utah.edu \\ F. Michael Christ \\ University of California \\ Los Angeles, CA 90024-1555 \\ christ@math.ucla.edu \\ THOMAS ENRIGHT \\ University of California, San Diego \\ La Jolla, CA 92093 \\ tenright@ucsd.edu
}

\author{
Nicholas ERcolani \\ University of Arizona \\ Tucson, AZ 85721 \\ ercolani@math.arizona.edu \\ R. FINN \\ Stanford University \\ Stanford, CA 94305 \\ finn@gauss.stanford.edu \\ VAughan F. R. Jones \\ University of California \\ Berkeley, CA 94720 \\ vfr@math.berkeley.edu \\ STEVEN KeRCKHOFF \\ Stanford University \\ Stanford, CA 94305 \\ spk@gauss.stanford.edu
}

\author{
C. C. MOORE \\ University of California \\ Berkeley, CA 94720
}

MARTIN SCHARLEMANN

University of California

Santa Barbara, CA 93106

mgscharl@henri.ucsb.edu

\author{
HAROLD STARK \\ University of California, San Diego \\ La Jolla, CA 92093
}

\section{SUPPORTING INSTITUTIONS}

UNIVERSITY OF ARIZONA
UNIVERSITY OF BRITISH COLUMBIA
CALIFORNIA INSTITUTE OF TECHNOLOGY
UNIVERSITY OF CALIFORNIA
MONTANA STATE UNIVERSITY
UNIVERSITY OF NEVADA, RENO
NEW MEXICO STATE UNIVERSITY
OREGON STATE UNIVERSITY

\author{
UNIVERSITY OF OREGON \\ UNIVERSITY OF SOUTHERN CALIFORNIA \\ STANFORD UNIVERSITY \\ UNIVERSITY OF HAWAII \\ UNIVERSITY OF TOKYO \\ UNIVERSITY OF UTAH \\ WASHINGTON STATE UNIVERSITY \\ UNIVERSITY OF WASHINGTON
}

The Supporting Institutions listed above contribute to the cost of publication of this Journal, but they are not owners or publishers and have no responsibility for its content or policies.

\begin{abstract}
Mathematical papers intended for publication in the Pacific Journal of Mathematics should be in typed form or offset-reproduced (not dittoed), double spaced with large margins. Please do not use built up fractions in the text of the manuscript. However, you may use them in the displayed equations. Underline Greek letters in red, German in green, and script in blue. The first paragraph must be capable of being used separately as a synopsis of the entire paper. In particular it should contain no bibliographic references. Please propose a heading for the odd numbered pages of less than 35 characters. Manuscripts, in triplicate, may be sent to any one of the editors. Please classify according to the 1991 Mathematics Subject Classification scheme which can be found in the December index volumes of Mathematical Reviews. Supply name and address of author to whom proofs should be sent. All other communications should be addressed to the managing editor, or Elaine Barth, University of California, Los Angeles, California 90024-1555.

There are page-charges associated with articles appearing in the Pacific Journal of Mathematics. These charges are expected to be paid by the author's University, Government Agency or Company. If the author or authors do not have access to such Institutional support these charges are waived. Single authors will receive 50 free reprints; joint authors will receive a total of 100 free reprints. Additional copies may be obtained at cost in multiples of 50 .
\end{abstract}

The Pacific Journal of Mathematics (ISSN 0030-8730) is published monthly except for July and August. Regular subscription rate: $\$ 190.00$ a year (10 issues). Special rate: $\$ 95.00$ a year to individual members of supporting institutions.

Subscriptions, orders for numbers issued in the last three calendar years, and changes of address should be sent to Pacific Journal of Mathematics, P.O. Box 969, Carmel Valley, CA 93924, U.S.A. Old back numbers obtainable from Kraus Periodicals Co., Route 100, Millwood, NY 10546.

The Pacific Journal of Mathematics at P.O. Box 969, Carmel Valley, CA 93924 (ISSN 0030-8730) is published monthly except for July and August. Second-class postage paid at Carmel Valley, California 93924, and additional mailing offices. Postmaster: send address changes to Pacific Journal of Mathematics, P.O. Box 969, Carmel Valley, CA 93924.

\section{PUBLISHED BY PACIFIC JOURNAL OF MATHEMATICS, A NON-PROFIT CORPORATION} Copyright (C) 1992 by Pacific Journal of Mathematics 


\section{PACIFIC JOURNAL OF MATHEMATICS}

Volume $156 \quad$ No. $2 \quad$ December 1992

Surfaces in the 3-dimensional Lorentz-Minkowski space satisfying 201 $\Delta x=A x+B$

Luis Alías, Angel FerRandez and Pascual LuCAS

Lie algebras of type $D_{4}$ over number fields

209

BRUCE ALLISON

Subsemigroups of completely simple semigroups

251

Anne Antonippillai and Francis PAStiJn

Studying links via closed braids. VI. A nonfiniteness theorem

JOAN Birman and William W. MENASCO

Minimal orbits at infinity in homogeneous spaces of nonpositive curvature

MARÍA J. DRUETTA

Generalized horseshoe maps and inverse limits

SARAH ElizABETH Holte

Determinantal criteria for transversality of morphisms

DAN LAKSOV and ROBERT SPEISER

Four dodecahedral spaces

PETER LORIMER

Semifree actions on spheres

MONICA NiCOLAU

Conformal deformations preserving the Gauss map

ENALDO VERGASTA

Hecke eigenforms and representation numbers of arbitrary rank lattices 371

LYNNE WALLING 